\title{
Collective viscous propulsion of a two-dimensional flotilla of Marangoni boats
}

Darren Crowdy ${ }^{1, \text { a) }}$

Department of Mathematics, Imperial College London, 180 Queen's Gate, London SW7 2AZ, U.K.

(Dated: 15 November 2020)

A closed-form solution is presented for the collective Marangoni-induced motion of a two-dimensional periodic array, or "flotilla", of Marangoni boats on deep water at zero Reynolds, capillary and surface Péclet numbers. The physical set-up is identical to the model of Marangoni propulsion proposed by Lauga \& Davis [J. Fluid Mech., 705, (2012)] but accounts now for interaction effects between boats, and in a simpler two-dimensional setting. The boats are modelled as identical thin floating strips each self-actuated by a trailing edge surfactant source that lowers the surface tension there according to a linear equation of state. The collective Marangoni propulsion speed of a flotilla of boats is found to be $(2 \pi \mu \delta)^{-1} \Delta \sigma \log \sec (\pi \delta / 2)$ where $\delta$ is the meniscus coverage fraction, $\mu$ is the subphase fluid viscosity and $\Delta \sigma$ is the surfactant-induced surface tension disparity across each boat. The theoretical result exemplifies the mechanism for collective rectilinear motion due to Marangoni convection caused by the diffusion of insoluble surfactant.

Keywords: Marangoni boat, camphor boat, viscous propulsion, active particle.

a) Electronic mail: d.crowdy@imperial.ac.uk 


\section{INTRODUCTION}

There has been much recent interest in the study of collective motion of self-driven organisms, particles and swimmers and their self-assembly into patterns, lattices or other dynamically emergent structures ${ }^{1-3}$. Self-organization on a free surface due to capillary interactions between floating objects has been a particularly active area ${ }^{4}$. Other challenges involving free surfaces include the physico-chemical design and analysis of self-propelled objects caused by chemical gradients where a non-uniformity in surface tension is responsible for motion. A commonly used device on free surfaces is the strategic spreading of surfactants to provoke a surface tension gradient, and a concomitant surface Marangoni stress, that can lead to locomotion. This phenomenon is familiar in natural biological settings ${ }^{5}$ and has also been exploited in synthetic situations ${ }^{2,3}$. Recent studies of "Marangoni surfers" ${ }^{6,7}$ exploit stresses associated with surface gradients in both temperature and surfactant concentration to generate motion.

Many effects can come into play in causing the motion of a single object, or the collective motion of many, on a free surface. The presence of surfactants usually alters the local surface tension value and these can be ejected by a particle or organism as a form of actuation. Those surfactants can diffuse along the surface, or into the bulk fluid, sublimate into the ambient gas, or be convected by fluid motions, thereby setting up a surface tension gradient resulting in a Marangoni stress at the interface. External stimulation mechanisms can also serve to alter the local surface tension in order to manipulate particles on free surfaces ${ }^{8-10}$.

Spatiotemporal collective motions involving "camphor boats" have been a topic of extensive investigation both experimentally and theoretically ${ }^{2,3,11}$. A camphor boat is an entity floating on a free surface where a camphor grain placed at some point on its intersection with the surface can locally reduce the surface tension there as the camphor invades the surface where it generally spreads, diffuses and sublimates. Multi-boat interactions are of current interest and early studies of two boat interactions ${ }^{12}$ demonstrated the possibility of phase locking of multiple boats into synchronized motion. Similar phenomena have been observed for oil droplets ${ }^{13}$ where induced convection rolls aid in providing a self-sustaining fuel source; in these experiments it was noticed that two approaching oil droplets can exhibit a synchronized motion with one droplet following the other. A homogeneous, or "uniform flow", state of many boats in an annular channel has been observed ${ }^{14-17}$ together with a 
"congested state" reminiscent of a traffic jam.

Significant effort has gone into trying to understand these observations using theoretical models. Most of the models are based on an equation of motion for each boat based on Newton's second law coupled with a reaction-diffusion equation for the camphor concentration which feeds back to alter the surface tension force appearing in the force imbalance causing motion of the boat ${ }^{11,16}$. As observed in a recent review ${ }^{3}$, few of these models properly account for hydrodynamic effects and, in particular, any flow in the bulk caused by surface Marangoni stresses which are an additional source of forces on the boats. Several attempts at visualizing the convection rolls in the bulk driven by the surface Marangoni stresses have been made m,15,18,19 $^{3}$ and the influence of these Marangoni effects is broadly acknowledged ${ }^{19,20}$. Recent work involving a camphor particle on the surface of a semi-infinite fluid proposes to fix an observed discrepancy in the rate of diffusion by assigning an effective diffusion coefficient taking account of the Marangoni effect ${ }^{20,21}$.

The importance of these often neglected Marangoni stresses also showed itself in a theoretical study of viscous Marangoni propulsion ${ }^{22}$. In that study a model problem was introduced comprising a single thin circular disc floating on a semi-infinite layer of viscous fluid and self-actuated by the setting up of a prescribed surfactant distribution on a segment of its surface. The model assumes the surfactant is insoluble and that the Reynolds number, capillary number and surface Péclet number are small so that locomotion is caused purely by the diffusion of the insoluble surfactant over the free surface. This diffusion changes the surface tension distribution producing a Marangoni-induced flow in the bulk which, in turn, induces a net motion of the floating disc by virtue of the requirement that, in such a low Reynolds number situation, the boat must be free of net force. An analytical expression for the net speed of propulsion is derived that is a function of the actuation profile ${ }^{22}$. One outcome of the investigation is that this propulsion speed can be quite different to that obtained by ignoring details of the Marangoni-induced flow altogether and estimating it instead based on a balance of forces between surface tension and the viscous drag on a forced particle.

The present article is concerned with multi-boat interactions with a view to gaining theoretical insights into the importance of Marangoni-induced flows in the various self-assembly scenarios surveyed above. It is shown here that the essential mechanism for collective propulsion can be exemplified in a simple two-dimensional model problem that is solvable in closed form when the Reynolds number, capillary number and surface Péclet number are negligible; 
these are the same assumptions used in the model of Lauga and Davis ${ }^{22}$. The new solution gives the speed $U_{0}$ of propulsion of a periodic array, or flotilla, of Marangoni boats to be

$$
U_{0}=\frac{\Delta \sigma}{2 \pi \mu}\left\{\frac{1}{\delta} \log \sec \left(\frac{\pi \delta}{2}\right)\right\}
$$

where $\delta=a / b$ is the meniscus coverage fraction, $\mu$ is the subphase fluid viscosity and $\Delta \sigma$

is the surfactant-induced surface tension disparity across each boat. The flow beneath the cotravelling flotilla is also describable explicitly. A parametric form of the streamfunction $\psi$ associated with the steady hydrodynamic flow in the region $y<0$ beneath the flotilla of Marangoni boats on $y=0$, in a frame of reference cotravelling with speed $U_{0}$, is

$$
\begin{aligned}
\psi(z, \bar{z}) & =\operatorname{Im}[(\bar{z}-z) f(z)], \quad z=x+\mathrm{i} y \\
z & =Z(\zeta)=+\frac{\mathrm{i} b}{\pi} \log \left\{-\frac{(\zeta-\alpha)(\zeta-1 / \alpha)}{(\zeta-\bar{\alpha})(\zeta-1 / \bar{\alpha})}\right\}, \quad \alpha=\mathrm{i} \tan \left(\frac{\pi \delta}{4}\right), \\
f(z) & =F(\zeta)=\frac{\Delta \sigma}{4 \mu \pi}\left(\frac{1}{\delta}\right) \log \left(\frac{\zeta-1 / \alpha}{\zeta-1 / \bar{\alpha}}\right),
\end{aligned}
$$

where the parameter $\zeta$ is any point in an upper-half unit disc in a parametric $\zeta$ plane as shown in Figure 2 and explained later.

The purpose of this article is to derive this analytical solution and study its characteristics: this is done in $\S \mathrm{II}-\mathrm{V}$. In $\S \mathrm{VI}$ we discuss how it might be used as a non-trivial base-state solution where additional physical effects can be incorporated perturbatively to understand how they affect the base-state propulsion speed (1).

\section{THE FLOTILLA MODEL}

A fluid of viscosity $\mu$ is assumed to occupy the lower half plane $y<0$ in a Cartesian $(x, y)$ plane as shown in Figure 1 and to be in the Stokes flow regime with incompressible velocity field $\mathbf{u}=(u, v)$. A spatially-periodic flotilla of identical "Marangoni boats" is situated on the fluid surface at $y=0$, each boat being a strip of length $2 L=2(b-a)$ with $a<b$, with centres which are distance $2 b$ apart. These identical boats will be actuated by a surfactant source at the left-hand side of each boat shown in Figure 1; this surfactant lowers the local value of the surface tension there causing the surface tension at the rear of any boat to be lower than that at the front. This surface tension disparity, $\Delta \sigma$ say, between the front and 


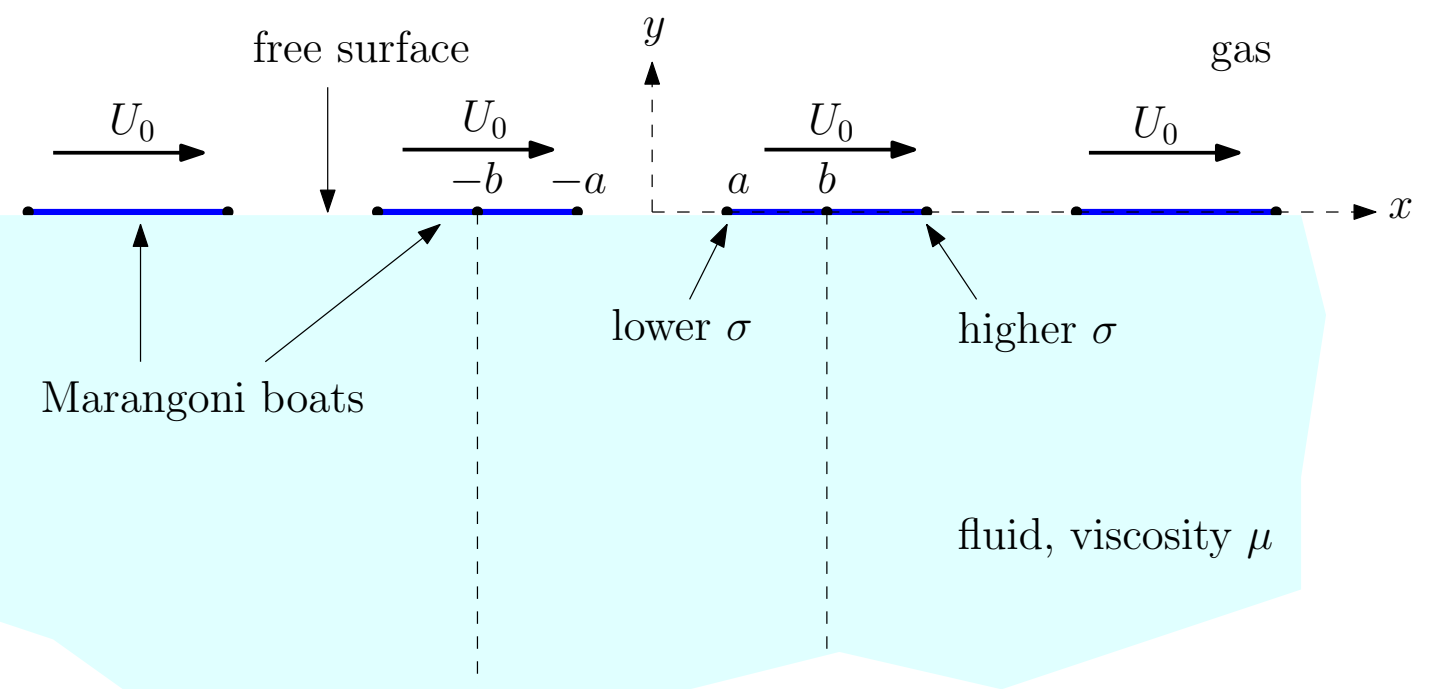

FIG. 1. A flotilla of identical Marangoni boats travelling collectively with steady speed $U_{0}$ to the right due to a surfactant-induced surface tension difference $\Delta \sigma$ fore and aft of each boat. The bulk fluid, which occupies the lower half plane and is of viscosity $\mu$, is in the Stokes flow regime. A constant pressure gas occupies the upper half plane.

rear of each boat will cause collective propulsion of the flotilla in the positive $x$ direction as indicated in Figure 1. It is the propulsion speed $U_{0}$ that will be determined from the condition that the boats are free of net force.

Halpern and Frenkel ${ }^{23}$ (see their appendix B) give a simple derivation of the following two-dimensional equation governing the evolution of insoluble surfactant with density $\Gamma(x, t)$ on an interface $y=\eta(x, t)$ :

$$
\frac{\partial}{\partial t}\left[\Gamma \sqrt{1+\eta_{x}^{2}}\right]+\frac{\partial}{\partial x}\left[\Gamma \tilde{u} \sqrt{1+\eta_{x}^{2}}\right]=D_{s} \frac{\partial}{\partial x}\left[\frac{1}{\sqrt{1+\eta_{x}^{2}}} \frac{\partial \Gamma}{\partial x}\right], \quad \eta_{x} \equiv \frac{\partial \eta}{\partial x}
$$

where $\tilde{u}$ denotes the fluid velocity component in the $x$ direction on the interface and $D_{s}$ is the surface diffusion coefficient. With a flat interface $\eta(x)=0$, and non-dimensionalizing lengths with respect to $L$, velocities with respect to the boat speed $U_{0}$ and time with respect to $L / U_{0}$, the non-dimensional form of $(3)$ is

$$
P e_{s}\left[\frac{\partial \Gamma}{\partial t}+\frac{\partial}{\partial x}(\Gamma \tilde{u})\right]=\frac{\partial^{2} \Gamma}{\partial x^{2}}, \quad P e_{s}=\frac{U_{0} L}{D_{s}}
$$

where all quantities are now taken to be non-dimensional and $P e_{s}$ is the surface Péclet 
number. In the limit $P e_{s} \rightarrow 0$ where surface diffusion dominates, equation (4) reduces to

$$
\frac{\partial^{2} \Gamma}{\partial x^{2}}=0
$$

To find a solution for a steadily-translating flotilla it is expedient to move to a frame of reference translating with the a priori unknown speed $U_{0}$. The associated fluid velocity field is $2 b$-periodic so it is enough to study the problem in the principal period window given by the semi-strip

$$
-b<x \leq b, \quad y \leq 0 .
$$

Under an assumption, to be discussed shortly, that a certain capillary number is negligible the interstitial meniscus portions between the boats can be taken to be flat and located on $y=0$. The fluid velocity as $y \rightarrow-\infty$ in the cotravelling frame is

$$
(u, v) \rightarrow\left(-U_{0}, 0\right), \quad \text { as } y \rightarrow-\infty .
$$

The surfactant distribution, which will be a function of $x$ only, can be found by solving (5),

$$
\Gamma(x)=A x+B,
$$

where $A$ and $B$ are constants of integration. They are chosen to ensure that the surfactant concentration has the imposed value at each end of a boat.

It is a common, especially when surfactant levels are moderate, to assume a linear equation of state ${ }^{22,24,25}$ relating the surfactant concentration $\Gamma(x)$ to the local surface tension value $\sigma$ :

$$
\sigma=\sigma_{0}-\beta \Gamma(x),
$$

where $\sigma_{0}$ is the clean-flow surface tension and $\beta=R T$, where $R$ is the gas constant and $T$ is absolute temperature, reflects the sensitivity of the surface tension to changes in the surfactant concentration. With such an assumption, and in view of (8), the interfacial surface tension $\sigma$ is a linear function of $x$. With the origin at the meniscus centre as in Figure 1, we can write

$$
\sigma=\Sigma(x)=\sigma_{1}-\frac{\Delta \sigma}{2 a} x, \quad|x|<a,
$$


where the constant $\sigma_{1}$ is the surface tension at the centre of the meniscus. It follows that

$$
\sigma=\left\{\begin{array}{l}
\sigma_{1}-\Delta \sigma / 2, \quad x=a(\text { aft }), \\
\sigma_{1}+\Delta \sigma / 2, \quad x=-a(\text { fore })
\end{array}\right.
$$

so the surface tension at the front of a boat exceeds that at its rear by a specified amount $\Delta \sigma$. Propulsion in the positive $x$ direction is expected. In this model the surfactant concentration values at the boat edges, and the parameter $\beta$, are lumped into the surface tension difference $\Delta \sigma$; only $\Delta \sigma$, and the linear profile (10), are pertinent to the model. It is important to emphasize that the linear profile (10) is not a postulated ansatz but emerges naturally as a solution of the surfactant evolution equation (4) at zero surface Péclet number together with the linear equation of state (9).

Let us assume that the region above the liquid layer is occupied by gas at uniform pressure $p_{g}$. Suppose the pressure in the fluid is denoted by

$$
p(x, y)=p_{0}+P(x, y)
$$

where $p_{0}$ is the average fluid pressure on the interface and $P(x, y)$ is the deviation from this average value. On each portion of the meniscus the stress induced by the non-uniform surface tension must be balanced by the fluid stress or $^{24,25}$

$$
[\mathbf{T} . \mathbf{n}]_{\text {gas }}^{\text {fluid }}=\sigma \kappa \mathbf{n}-\nabla_{s} \sigma, \quad \mathbf{T} \equiv-p \mathbf{I}+\mu\left[\nabla \mathbf{u}+(\nabla \mathbf{u})^{T}\right]
$$

where $\mathbf{T}$ is the fluid stress tensor in either phase, $[\mathbf{T} . \mathbf{n}]_{\text {gas }}^{\text {fluid }}$ denotes the jump in the bracketed quantity between its limit approached from the fluid side and from the gas side, $\kappa$ is the curvature of the surface and $\nabla_{s}=(\mathbf{I}-\mathbf{n n}) . \nabla$ denotes the surface gradient on the interface having unit normal $\mathbf{n}$ directed into the fluid. The tangential projection of the boundary condition (13) along the interface gives the Marangoni stress balance

$$
\mu \mathbf{t} .\left[\nabla \mathbf{u}+(\nabla \mathbf{u})^{T}\right] . \mathbf{n}=-\frac{\partial \sigma}{\partial s},
$$

where $s$ denotes arclength along the boundary taken such that $s$ increases with the fluid region on the left. On use of (11) and (14) the boat speed $U_{0}$ will scale with the jump in 
surface tension $\Delta \sigma$ across it:

$$
U_{0} \sim \frac{\Delta \sigma}{\mu}
$$

The normal component of (13) is

$$
-\left(p_{0}-p_{g}\right)-P(x, y)+\mu \mathbf{n} \cdot\left[\nabla \mathbf{u}+(\nabla \mathbf{u})^{T}\right] . \mathbf{n}=\kappa\left(\sigma_{1}-\frac{\Delta \sigma}{2 a} x\right) .
$$

If lengths are non-dimensionalized with respect to $L$, and the fluid pressure disturbance $P(x, y)$ with respect to $\mu U_{0} / L,(16)$ can be written as

$$
-\frac{\left(p_{0}-p_{g}\right) L}{\sigma_{1}}+\mathrm{Ca}\left[-\hat{P}(x, y)+\mathbf{n} \cdot\left[\nabla \hat{\mathbf{u}}+(\nabla \hat{\mathbf{u}})^{T}\right] . \mathbf{n}\right]=\hat{\kappa}\left(1-\frac{\Delta \sigma}{\sigma_{1}} \frac{L}{2 a} \hat{x}\right)
$$

where hats denote non-dimensional quantities and the capillary number is defined as

$$
\mathrm{Ca}=\frac{\mu U_{0}}{\sigma_{1}}
$$

On use of (15) the final term of (17) can be seen to be of order Ca since

$$
\frac{\Delta \sigma}{\sigma_{1}} \sim \mathrm{Ca}
$$

Therefore if $\mathrm{Ca} \ll 1$ the dominant balance in the normal stress condition (17) is

$$
-\frac{\left(p_{0}-p_{g}\right) L}{\sigma_{1}}=\hat{\kappa}
$$

A constant curvature interface is therefore a consistent solution in this small capillary number limit. For the flat interface of interest here, $\hat{\kappa}=0$ and $p_{0}=p_{g}$ (which, without loss of generality, we can set to zero). For such an interface to be a consistent equilibrium it must be a streamline of the flow.

In summary, in the small capillary number limit the line $y=0$ is a streamline with the Marangoni stress balance (14) holding on each meniscus portion of this line. On any boat the no-slip condition

$$
(u, v)=(0,0)
$$

holds. At the edges of each boat the fluid velocities must be continuous but integrable 
singularities of the fluid stress are expected. The propulsion speed $U_{0}$ will be set by the condition of zero net force on each boat.

\section{COMPLEX VARIABLE FORMULATION}

The challenge now is to solve a periodic, two-dimensional Stokes flow problem in the lower half-plane $y<0$ with the mixed boundary conditions on $y=0$ just discussed. It is convenient in this section to proceed using dimensional variables. The assumed incompressible Stokes flow in the bulk means that a complex variable representation of a biharmonic streamfunction $\psi(z, \bar{z})$ can be introduced given by $^{26,27}$

$$
\psi(z, \bar{z})=\operatorname{Im}[\bar{z} f(z)+g(z)], \quad(u, v)=\left(\frac{\partial \psi}{\partial y},-\frac{\partial \psi}{\partial x}\right), \quad u-\mathrm{i} v=2 \mathrm{i} \frac{\partial \psi}{\partial z}
$$

where $f(z)$ and $g(z)$ are two functions of $z=x+\mathrm{i} y$, having the dimensions of a velocity, that are analytic in the lower-half plane except possibly on $y=0$ and at infinity. Under this change of variables, $(x, y) \mapsto(z, \bar{z})$, it is easy to check that

$$
\frac{\partial}{\partial z}=\frac{1}{2}\left[\frac{\partial}{\partial x}-\mathrm{i} \frac{\partial}{\partial y}\right], \quad \frac{\partial}{\partial \bar{z}}=\frac{1}{2}\left[\frac{\partial}{\partial x}+\mathrm{i} \frac{\partial}{\partial y}\right]
$$

From the Stokes equations, and using (23), it is a straightforward exercise to verify that the fluid pressure $P$, the vorticity $\omega=-\nabla^{2} \psi=-4 \partial^{2} \psi / \partial z \partial \bar{z}$ and the fluid rate-of-strain tensor $e_{i j}=(1 / 2)\left(\partial u_{i} / \partial x_{j}+\partial u_{j} / \partial x_{i}\right)$ are related to $f(z)$ and $g(z)$ through

$$
4 f^{\prime}(z)=\frac{P}{\mu}-\mathrm{i} \omega, \quad u-\mathrm{i} v=-\overline{f(z)}+\bar{z} f^{\prime}(z)+g^{\prime}(z), \quad e_{11}+\mathrm{i} e_{12}=z \overline{f^{\prime \prime}(z)}+\overline{g^{\prime \prime}(z)}
$$

where primes denote differentiation with respect to $z$. The appendix gives a derivation of these fundamental relations.

There is an additive degree of freedom in the choice of $f(z)$ and $g^{\prime}(z)$ since the transformations $f(z) \mapsto f(z)+c$ and $g^{\prime}(z) \mapsto g^{\prime}(z)+\bar{c}$ where $c$ is a complex constant can be seen not to affect the complex velocity field $u-\mathrm{i} v$ given in (24). This degree of freedom will be specified later. It is also important to note that although the velocity and stress fields in the fluid are $2 b$-periodic the functions $f(z)$ and $g(z)$ do not necessarily share this property. 
On inspection of (22) it can be checked that the choice

$$
g(z)=-z f(z)
$$

ensures that $\psi=0$ on $y=0$, where $\bar{z}=z$, and renders it a streamline; this choice turns out to be consistent with the far-field requirements and force-free condition on the boat as will be verified. The complex velocity field, with $g(z)$ given by $(25)$, is

$$
u-\mathrm{i} v=-\overline{f(z)}+\bar{z} f^{\prime}(z)+g^{\prime}(z)=-\overline{f(z)}+(\bar{z}-z) f^{\prime}(z)-f(z) .
$$

The notation $\operatorname{Re}[f(z)]$ and $\operatorname{Im}[f(z)]$ is used to denote the real and imaginary parts of $f(z)$, respectively. Since we require the velocity field to be $2 b$-periodic it is clear from (26) that $\operatorname{Re}[f(z)]$, and its derivative $f^{\prime}(z)$, must be $2 b$-periodic; there is no periodicity requirement on $\operatorname{Im}[f(z)]$. It is also noted from the first relation in $(24)$ that the periodicity of $f^{\prime}(z)$ ensures that the fluid pressure field is similarly periodic.

On $y=0$, where $\bar{z}=z=x$, it is found from (26) that

$$
u-\mathrm{i} v=-f(z)-\overline{f(z)}=-2 \operatorname{Re}[f(z)]
$$

The boundary condition (21) then implies that on a boat,

$$
\operatorname{Re}[f(z)]=0, \quad a<|x|<b, \quad y=0 .
$$

It is clear from (7) and (26) that $f(z)$ must also satisfy the far-field condition

$$
f(z) \sim \frac{U_{0}}{2}+\mathrm{i} f_{\infty}+\mathcal{O}(1 / z), \quad \text { as } y \rightarrow-\infty
$$

where $f_{\infty}$ is some real constant.

The left hand side of (14) is the tangential fluid stress on any boundary of the fluid region. In the appendix it is shown that the complex form of the total stress exerted by the fluid on the boundary is

$$
-2 \mu \mathrm{i} \frac{d H}{d s}, \quad H(z, \bar{z})=f(z)+z \overline{f^{\prime}(z)}+\overline{g^{\prime}(z)}
$$

where we have used the fact that the complex form of the unit tangent is $d z / d s$ with the 
corresponding complex unit normal vector directed into the fluid given by $\mathrm{i} d z / d s$. The complex form of the tangential stress condition (14) on the meniscus is therefore

$$
\operatorname{Re}\left[\overline{\frac{d z}{d s}}\left(-2 \mu \mathrm{i} \frac{d H}{d s}\right)\right]=-\frac{\partial \sigma}{\partial s}, \quad|x|<a, \quad y=0
$$

Since we can take $s=-x$ with $z=x$ so that $d z / d s=-1$ on the meniscus, this is

$$
\operatorname{Re}\left[2 \mu \mathrm{i} \frac{d H}{d x}\right]=-\frac{\partial \sigma}{\partial x}=\frac{\Delta \sigma}{2 a}, \quad|x|<a, \quad y=0
$$

where we have used (10); recall that $s$ must increase with the fluid region on the left. On integrating (32) with respect to $x$ we find

$$
\operatorname{Im}[H]=-\left(\frac{\Delta \sigma}{4 \mu a}\right) x, \quad \text { on } \quad|x|<a, \quad y=0,
$$

where we have set an integration constant to vanish; this is allowable by the aforementioned additive degree of freedom in the choice of $f(z)$ and $g^{\prime}(z)$. But with the choice (25),

$$
H=f(z)-\overline{f(z)}=2 \mathrm{i} \operatorname{Im}[f(z)], \quad \text { on } \quad y=0
$$

Therefore, combining (33) and (34), on the meniscus,

$$
\operatorname{Im}[f(z)]=-\left(\frac{\Delta \sigma}{8 \mu a}\right) x, \quad \text { on }|x|<a, \quad y=0
$$

It is now demonstrated that the condition that each boat is free of net force is ensured by a requirement that $\operatorname{Im}[f(z)]$ is $2 b$-periodic. Let us consider the boat occupying the interval $[a, 2 b-a]$; see Figure 2. The force on it due to the surface tension difference caused by the presence of surfactant is

$$
\Sigma(2 b-a)-\Sigma(a)=+\Delta \sigma
$$

On integrating the fluid stress (30) over this boat the total viscous stress on it is

$$
-2 \mu \mathrm{i} \int_{\text {fore }}^{\mathrm{aft}} \frac{d H}{d s} d s=2 \mu \mathrm{i}\left[\left.H\right|_{z=2 b-a}-\left.H\right|_{z=a}\right]
$$

where we recall again that arclength increases with the fluid region to the left and Figures 1 
and 2 remind us that $z=2 b-a$ corresponds to the front of the boat and $z=a$ corresponds to its rear. Now (34) holds everywhere on $y=0$ implying that the total viscous stress on the boat is

$$
2 \mu \mathrm{i} \times 2 \mathrm{i} \operatorname{Im}[f(2 b-a)-f(a)] .
$$

If we now insist that $\operatorname{Im}[f(z)]$ is $2 b$-periodic then $(38)$ is

$$
-4 \mu \operatorname{Im}[f(-a)-f(a)]=-4 \mu \times\left[\frac{\Delta \sigma}{8 \mu}-\left(-\frac{\Delta \sigma}{8 \mu}\right)\right]=-\Delta \sigma,
$$

where we have used (35). The net force on the boat - that is, the sum of (36) and (39) - is then zero as required.

From (24) and (28) it is easy to check that $P=0$ on any boat so the normal pressure forces are in balance and it follows that the net torque on each boat vanishes.

Therefore, to satisfy all the problem requirements, it is necessary to seek an analytic function $f(z)$ which is $2 b$-periodic, i.e.,

$$
f(z+2 b)=f(z)
$$

Before proceeding with a derivation of the flotilla solution it is worth remarking on the single-boat version of this problem. Suppose a single boat occupies the interval $-L<x<L$ on $y=0$ so that $x<-L$ and $x>L$ are two infinite meniscus portions. The only admissible solution of (5) for which $\Gamma(x)$ does not diverge in the far field $|x| \rightarrow \infty$ is $\Gamma(x)=\Gamma_{+}$for $x>L$ and $\Gamma(x)=\Gamma_{-}$for $x<-L$ where $\Gamma_{ \pm}$are constants. Consequently, according to (9), the surface tensions on the menisci fore and aft of the boat will also be constant. If these constants are different, by an amount $\Delta \sigma$ say, there will certainly be a net surface tension force across the boat, but there will be no surface tension gradients and, hence, no Marangoni-induced flow. With no Marangoni-induced viscous drag to balance the net surface tension force no solution to this single-boat version of this two-dimensional pure diffusion-driven Marangoni propulsion problem can exist. Moreover, in two dimensions, it is known that the solution to the "dragging problem" - the flow caused by dragging the boat with a net force $\Delta \sigma$ through the viscous fluid - does not exist due to the Stokes paradox. Thus even an approximation of the boat speed induced by the surface tension disparity, akin to that computed by Lauga \& Davis ${ }^{22}$ for their single-boat problem in three dimensions, is 
not available in this two-dimensional single-boat analogue. In view of such obstructions, it is easy to overlook the possibility of the existence of solutions to the periodic version of this two-dimensional problem, i.e., the flotilla scenario studied here.

\section{SOLUTION BY CONFORMAL MAPPING}

To complete the solution for the flotilla it will now be shown that an analytic function $f(z)$ with all the properties specified above can be found. To do so it is expedient to introduce the modified analytic function

$$
h(z)=\mathrm{i} f(z)-\left(\frac{\Delta \sigma}{8 \mu a}\right) z .
$$

If $f(z)$ is $2 b$-periodic the function $h(z)$ will be quasi-periodic owing to the term proportional to $z$ in its definition (41). Let us now consider the boundary conditions on $h(z)$ induced by those on $f(z)$ established above. On the boat,

$$
\operatorname{Im}[h(z)]=\operatorname{Re}[f(z)]=0, \quad \text { on } \quad a<|x|<b, \quad y=0 .
$$

On the meniscus,

$$
\operatorname{Re}[h(z)]=-\operatorname{Im}[f(z)]-\left(\frac{\Delta \sigma}{8 \mu a}\right) x=0, \quad \text { on }|x|<a, \quad y=0
$$

where we have used (35). As $y \rightarrow-\infty$, and on use of (29), we note that

$$
h(z) \sim-\left(\frac{\Delta \sigma}{8 \mu a}\right) z+\frac{\mathrm{i} U_{0}}{2}, \quad \text { as } y \rightarrow-\infty .
$$

In particular, this implies

$$
\operatorname{Im}[h(z)] \sim-\dot{\gamma}(y-\lambda),
$$

where

$$
\dot{\gamma} \equiv\left(\frac{\Delta \sigma}{8 \mu a}\right) \quad \dot{\gamma} \lambda=\frac{U_{0}}{2}
$$


To summarize, we seek to find the quasi-periodic lower analytic function $h(z)$ with

$$
h(z) \sim-\dot{\gamma}(z-\mathrm{i} \lambda), \quad \text { as } y \rightarrow-\infty
$$

with mixed boundary conditions

$$
\begin{aligned}
& \operatorname{Im}[h(z)]=0, \quad \text { on } a<|x|<b, \quad y=0, \quad \text { (boat), } \\
& \operatorname{Re}[h(z)]=0, \quad \text { on }|x|<a, \quad y=0, \quad \text { (meniscus). }
\end{aligned}
$$

This turns out to be a standard mixed boundary value problem that arises in many different areas of mathematical physics. Fluid dynamicists interested in superhydrophobic surfaces might recognize it as relevant to modelling longitudinal shear flow over a periodic array of unidirectional no-shear slots in an otherwise no-slip surface ${ }^{28,29}$. In that application the imaginary part of the analytic function $h(z)$ is $w(x, y)$, the longitudinal fluid velocity parallel to the no-shear slots with simple shear $w(x, y) \rightarrow-\dot{\gamma}(y-\lambda)$ in the far-field as $y \rightarrow-\infty$ just as in (45). In that context the parameter $\lambda$ has the interpretation of a hydrodynamic slip length ${ }^{28,29}$; we will see below that essentially the same quantity furnishes the sought-after propagation speed $U_{0}$.

Philip ${ }^{28}$ uses two Schwarz-Christoffel mappings to derive a solution to this paradigmatic mixed boundary value problem, but he was certainly not the first to consider it ${ }^{30,31}$, nor does he provide any details of his solution derivation. The author ${ }^{29}$ rederived the solution using a novel method based on conformal slit mappings, an approach that affords a ready extension of the analytical solutions to more complicated geometries ${ }^{32}$. It is this form of the solution that will be used here, not least because it involves only log-rational functions and it avoids complications associated with picking branches of a multivalued $\cos ^{-1}$ function that appears in Philip's form of the same solution. The author's solution, adjusted from the original presentation ${ }^{29}$ so that the fluid occupies the lower half plane, is given in terms of a parametric variable $\zeta$ taking values in the upper-half unit disc as shown in Figure 2. It is

$$
\begin{gathered}
z=Z(\zeta) \equiv+\frac{\mathrm{i} b}{\pi} \log \left[-\frac{(\zeta-\alpha)(\zeta-1 / \alpha)}{(\zeta-\bar{\alpha})(\zeta-1 / \bar{\alpha})}\right], \\
h(z)=\mathcal{H}(\zeta) \equiv-\frac{\mathrm{i} b \dot{\gamma}}{\pi} \log \left[-\frac{(\zeta-\alpha)(\zeta-1 / \bar{\alpha})}{(\zeta-\bar{\alpha})(\zeta-1 / \alpha)}\right],
\end{gathered}
$$




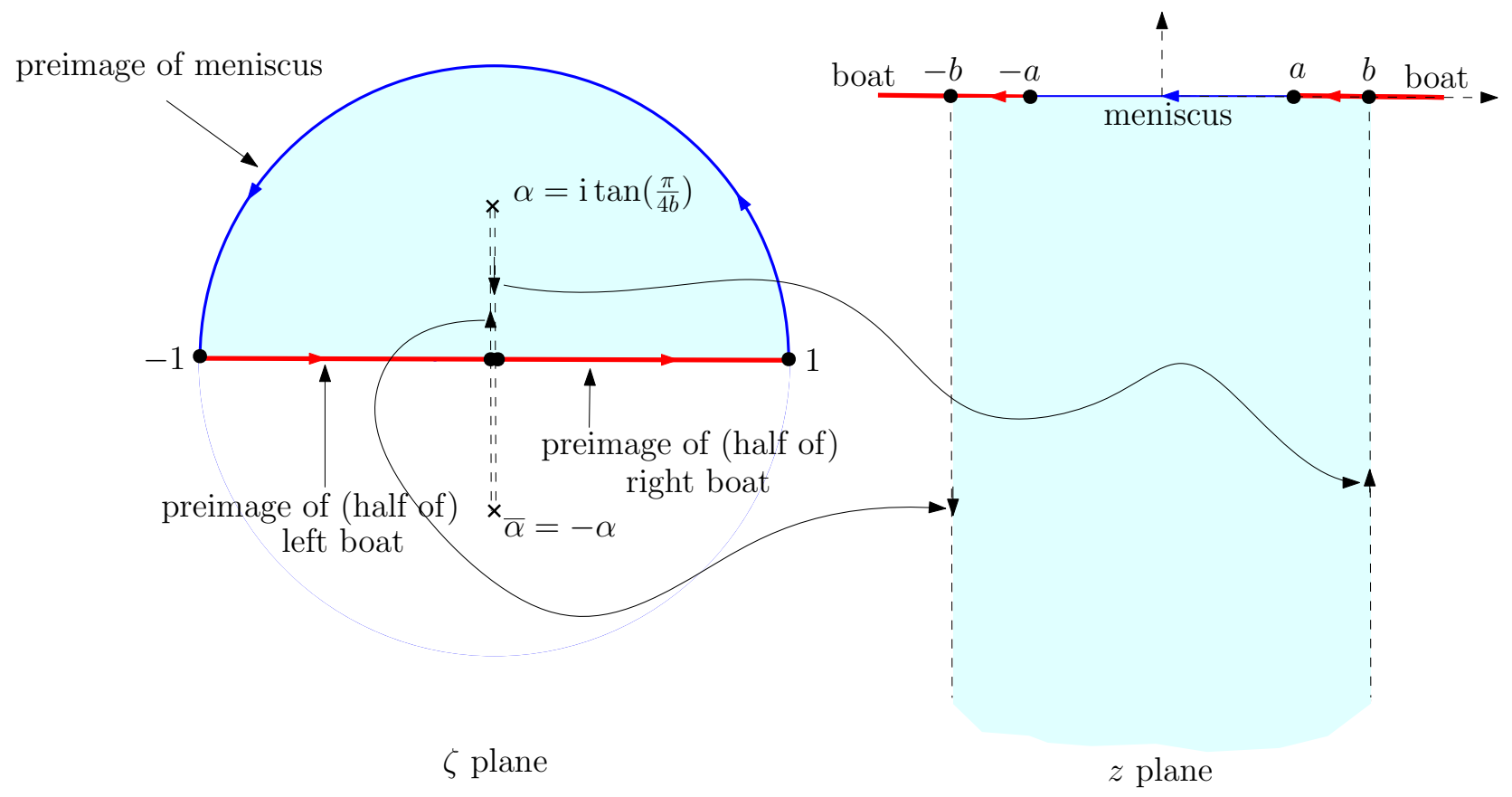

FIG. 2. The upper half disc in the $\zeta$ plane corresponds, under the conformal mapping $Z(\zeta)$ in (50), to a single period window in the physical $z$ plane. The two sides of a logarithmic branch cut between $\pm \alpha$ in the $\zeta$ plane map to the two edges of the period window.

where

$$
\alpha=\mathrm{i} \tan \left(\frac{\pi a}{4 b}\right)
$$

The function $f(z)$, written as a function of $\zeta$, now follows from (50) and (41):

$$
f(z)=F(\zeta)=\frac{\Delta \sigma}{4 \mu \pi}\left(\frac{b}{a}\right) \log \left(\frac{\zeta-1 / \alpha}{\zeta-1 / \bar{\alpha}}\right)
$$

where a branch cut joining the two logarithmic branch points at $1 / \alpha$ and $1 / \bar{\alpha}$ outside the unit disc can be selected along the imaginary $\zeta$-axis through the point at infinity. The required speed $U_{0}$ follows from (29) and (52):

$$
\frac{U_{0}}{2}=\lim _{y \rightarrow-\infty} \operatorname{Re}[f(z)]=\lim _{\zeta \rightarrow \alpha} \operatorname{Re}[F(\zeta)]=\frac{\Delta \sigma}{4 \mu \pi}\left(\frac{b}{a}\right) \log \left|\frac{\alpha-1 / \alpha}{\alpha-1 / \bar{\alpha}}\right|
$$

On use of (51), and some trigonometric identities, (53) leads to the final result (1) where we have introduced the meniscus coverage fraction $\delta=a / b$. The solution is complete and is summarized in (2).

It can be verified directly that (52), with $\zeta$ related to $z$ according to (50), satisfies all 
the required conditions of the problem. The periodicity (40) is confirmed by noticing, using the conformal mapping $Z(\zeta)$ depicted in Figure 2, that moving from the principal period window to a neighbouring one corresponds to making a complete continuous loop, either clockwise or anticlockwise, around the point $\zeta=\alpha$ and that $f(z)=F(\zeta)$ is unchanged by this action.

If desired it is just a matter of some algebra to eliminate the parameter $\zeta$ in $(2)$ - that is, to find the inverse function to $z=Z(\zeta)$ - to produce an explicit expression for $f(z)$ in terms of $z$. The resulting form involves trigonometric and inverse trigonometric functions ${ }^{28}$. This step is not necessary, however, and we proceed without it because it introduces square root branch points which can be cumbersome to deal with numerically. These branch points have been conveniently avoided, or "uniformized", by the parametric solution $(2)^{29,32}$.

\section{CHARACTERIZATION OF THE SOLUTIONS}

Figure 3 shows a graph of the normalized propagation speed

$$
\frac{2 \pi \mu U_{0}}{\Delta \sigma}=\frac{1}{\delta} \log \sec \left(\frac{\pi \delta}{2}\right)
$$

as a function of the meniscus coverage fraction $\delta=a / b$. As $\delta \rightarrow 0$ the meniscus disappears leaving no propulsion mechanism and resulting, as expected, in $U_{0} \rightarrow 0$. The propagation speed $U_{0}$ increases monotonically with $\delta$ and diverges logarithmically as $\delta \rightarrow 1$, indeed, if $\delta=1-\epsilon$ then as $\epsilon \rightarrow 0$,

$$
\frac{2 \pi \mu U_{0}}{\Delta \sigma}=-\frac{1}{1-\epsilon} \log \sin \left(\frac{\pi \epsilon}{2}\right) \sim-\log \epsilon
$$

This divergence can be understood using an effective singularity interpretation. As the meniscus coverage ratio $\delta \rightarrow 1$, for any fixed value of $b$, the length of each boat becomes vanishingly small compared to $b$. Consequently, the non-zero net surface tension force $\Delta \sigma$

in the positive $x$-direction associated with the surfactant density difference fore and aft of each boat is exerted over a vanishingly small distance; the net effect of this surface tension disparity over the boat is therefore close to that of a two-dimensional point force, or Stokeslet, singularity. To produce a zero net force on the boat, the compensating Marangoni flow must therefore be close to that generated by a Stokeslet of opposite strength. It is well-known that 
the velocity associated with a two-dimensional Stokeslet diverges logarithmically in the farfield; this is the underlying reason for the so-called Stokes paradox. With this interpretation the logarithmic divergence of the boat speed in the $\delta \rightarrow 1$ limit is to be expected.

The singularity in propulsion speed in the small-boat limit $\delta \rightarrow 1$ is likely to be difficult to realize in practice since it is more likely that, as the boat vanishes, so too will the driving mechanism. Consequently, suppose the surface tension jump $\Delta \sigma$ across a Marangoni boat is a function of its length, e.g., $\Delta \sigma \propto(1-\delta)^{\chi}$ for $\chi \geq 0$ so that $\Delta \sigma \rightarrow 0$ as $\delta \rightarrow 1$; the case $\chi=1$ would correspond to the surface tension jump across a boat being proportional to its length. Within such a model, for any $\chi>0$ the surface tension jump $\Delta \sigma$ across the boat vanishes with the boat length as $\delta \rightarrow 1$ and, according to (54), so too will the associated propulsion speed $U_{0}$. This is one way to regularize the unbounded propulsion speed in the small-boat limit $\delta \rightarrow 1$.

The single-boat scenario discussed at the end of $\S$ III corresponds to fixing the half-boat length $L=b-a$ and letting the half-period $b \rightarrow \infty$. It is clear that $\delta=1-L / b$ implying, on setting $\epsilon=L / b$ and using (55), that

$$
\frac{2 \pi \mu U_{0}}{\Delta \sigma} \sim \log (b / L), \quad \text { as } b \rightarrow \infty
$$

The speed $U_{0}$ diverges in the single-boat limit $b \rightarrow \infty$ which is consistent with the conclusion made earlier that the solution to the single-boat problem does not exist. The new periodic solutions found here for $b<\infty$ can therefore be viewed as a "regularization" of this singular single-boat limit. It is interesting to see how periodicity acts here as a regularization mechanism.

The streamfunction in the cotravelling frame is

$$
\psi(z, \bar{z})=\operatorname{Im}[(\bar{z}-z) f(z)]=\operatorname{Im}[-2 \mathrm{i} y f(z)]=-2 y \operatorname{Re}[f(z)] .
$$

The instantaneous streamfunction in the fixed non-moving frame is easily obtained by adding $U_{0} y$ to this. Plotting streamlines, which are the contours of these streamfunctions, is a simple matter given the explicit form of $f(z)$ in (2). Typical streamline patterns for $a=0.25,0.5$ and $a=0.9$ with $b=1$ are shown in Figure 4 : on the left the streamlines in the cotravelling frame are shown and, on the right, the instantaneous streamlines in the fixed frame with 


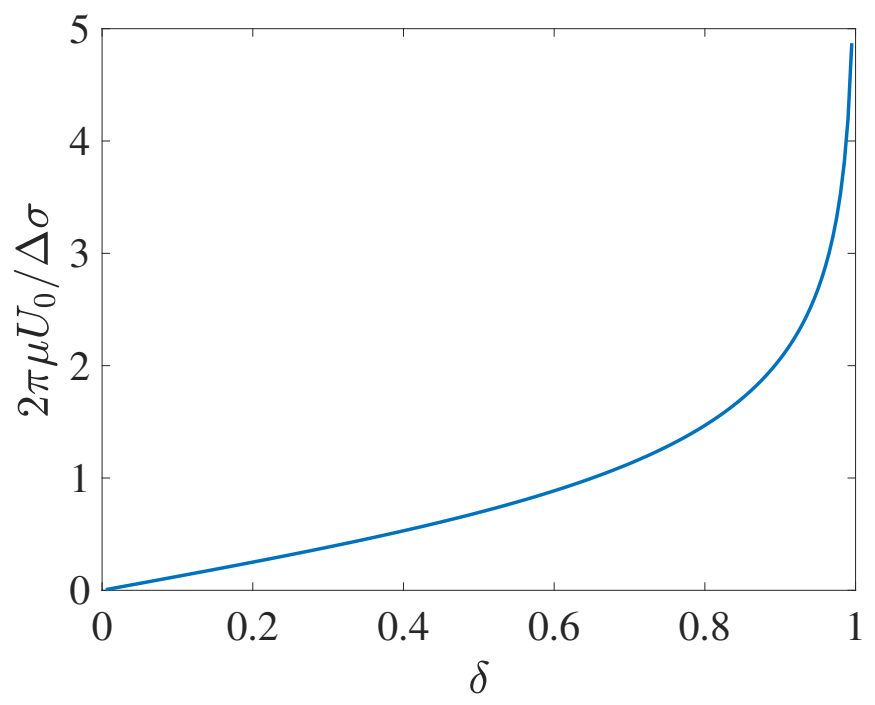

FIG. 3. The normalized propagation speed $2 \pi \mu U_{0} / \Delta \sigma$ as a function of the meniscus coverage fraction $\delta$. The speed diverges logarithmically as $\delta \rightarrow 1$ when the boats are small compared to their separation.

the boats at the same locations. These images are produced by plotting the streamlines in the upper half unit $\zeta$ disc, which is conveniently a bounded domain, and then pushing those streamlines forward into the physical $z$ domain using the mapping function $Z(\zeta)$ in (50). These streamline plots are qualitatively consistent with those already observed experimentally and theoretically by other authors in the single entity scenario $3,15,18,19$.

In the fixed frame two distinct eddy structures can be seen in each period window: one largely associated with the meniscus and another associated with the boat. For $\delta$ approximately in the interval $[0,0.5]$ these two eddies per period remain predominantly underneath the meniscus and underneath the boat respectively. There are two critical points on the meniscus at $\left( \pm x_{s}, 0\right)$ where the separatrix streamlines separating two recirculating eddies meet the meniscus. These critical points, which are stagnation points, remain close to the edges of the boat for $\delta$ roughly in the interval $[0,0.5]$. It is straightforward to manipulate the explicit solution (50) and (2) to find that they are located on the meniscus at $\left( \pm x_{s}, 0\right)$ where

$$
x_{s}=+\frac{\mathrm{i} b}{\pi} \log \left[-\frac{\left(\zeta_{s}-\alpha\right)\left(\zeta_{s}-1 / \alpha\right)}{\left(\zeta_{s}-\bar{\alpha}\right)\left(\zeta_{s}-1 / \bar{\alpha}\right)}\right], \quad \zeta_{s}=e^{\mathrm{i} \theta_{s}}, \quad \alpha=\mathrm{i} \tan \left(\frac{\pi \delta}{4}\right)
$$



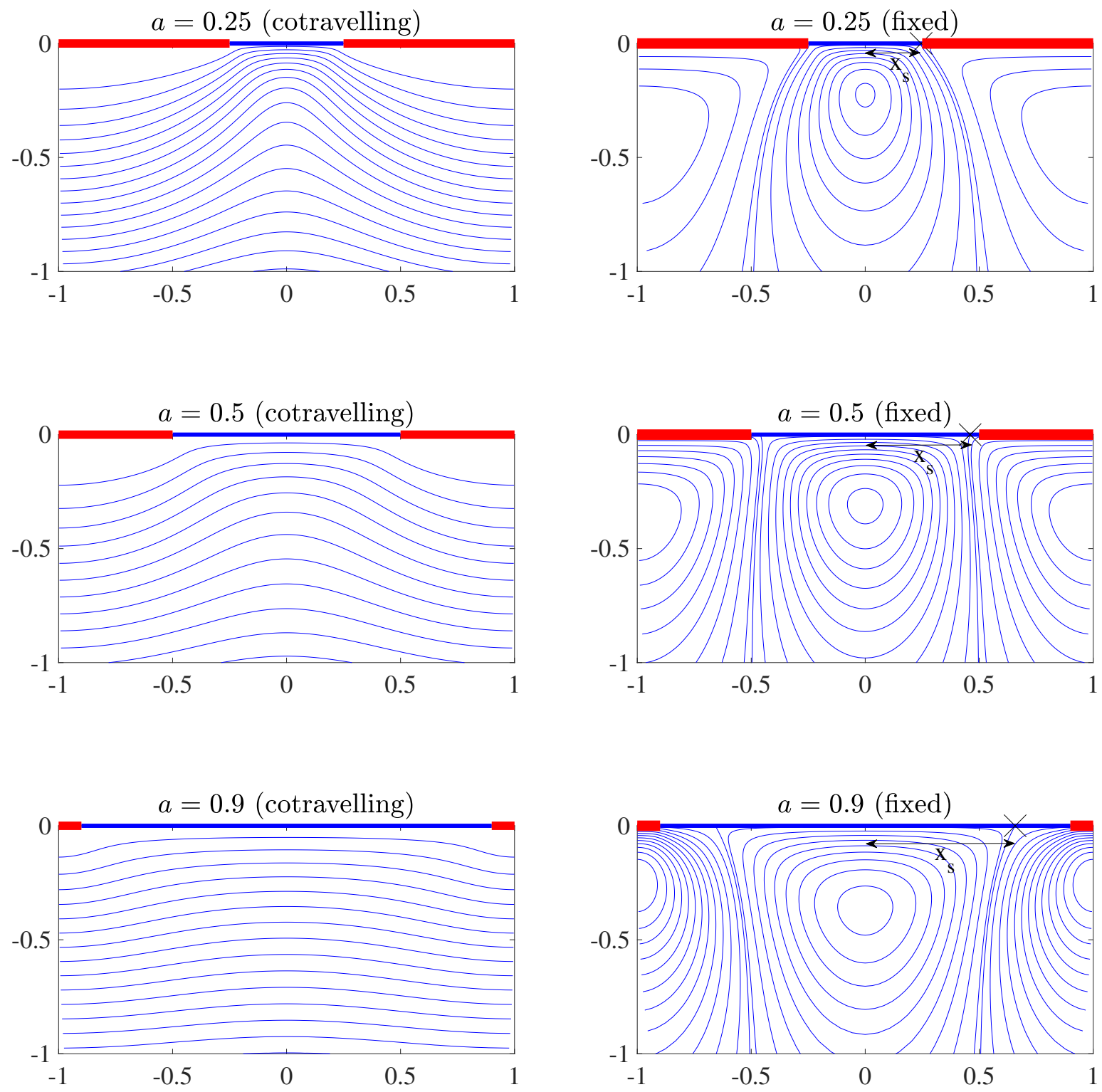

FIG. 4. Streamlines in the cotravelling frame (left column) and instantaneous streamlines in the fixed frame (right column) for $b=1$ and $a=0.25,0.5$ and 0.9. The boats have infinitesimal thickness but are shown by thick lines to distinguish them from the meniscus occupying $(-a, a)$. On the right, separatrix streamlines between recirculating eddies reach the meniscus at $\left( \pm x_{s}, 0\right)$.

where

$$
\theta_{s}=\sin ^{-1}\left(\frac{\sin (\pi \delta / 2)}{1+\cos ^{2}(\pi \delta / 2)}\right)
$$

A graph of $x_{s} / b$ against $\delta$ is shown in Figure 5 and shows that only for sufficiently small boats, i.e. for $\delta$ approximately in the interval $[0.5,1)$, do the critical points move significantly 


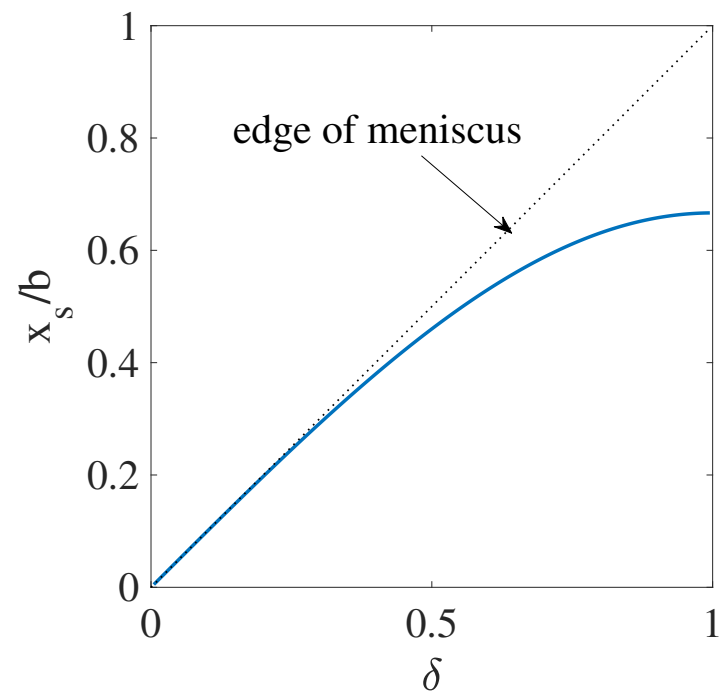

FIG. 5. Normalized critical point location $x_{s} / b$ as a function of $\delta$. The critical points remain close to the edge of the meniscus for $\delta$ approximately in the range $[0,0.5]$.

away from the edges of the boats towards the centre of the meniscus, but they never get too close to it. It would be interesting to examine the influence of these critical points between the recirculating eddy regions as more physical effects, such as advection, solubility and sublimation, are incorporated. This might be done within a perturbative framework based on these exact solutions as discussed in the next section.

\section{DISCUSSION}

The analytical solutions found here are expected to be important not only in exemplifying a basic mechanism of Marangoni propulsion driven by diffusion of insoluble surfactant, but as a non-trivial base state on which other physical effects can be added within a perturbative framework. This includes weak solubility and sorption kinetics, small Péclet number effects (both surface and bulk), small capillary number effects and meniscus deflection, small inertia effects and unsteadiness either in the surfactant evolution equation, or the hydrodynamics, or both. Even though the model is two-dimensional, examining how each of these affects the propulsion speed (1) should nevertheless be theoretically enlightening given all the different possible physical mechanisms in play, many of which can reasonably be expected to have qualitatively similar effects in a more realistic, but less analytically tractable, threedimensional geometry. One of the reasons we have presented our solutions as functions of the 
parametric variable $\zeta$ is that it affords a convenient way to add in new effects perturbatively as demonstrated in recent studies of other problems ${ }^{33,34}$.

It has already been discussed how the parameter $\Delta \sigma$ might be taken to be a function of the boat length but, within this quasi-steady model of a Stokes flow driven by fast surface diffusion, this parameter can also be made time-dependent - without affecting the solution herein to the Stokes flow problem - with its time evolution perhaps determined by some other prescription associated with the specifics of the boat driving mechanism.

The fundamental propulsion mechanism here has been diffusion of insoluble surfactant. Other mechanisms, such as capillary forces due to menisci with non-zero curvature ${ }^{4}$, are likely amenable to a similar mathematical approach and warrant further investigation. Also of interest is to examine how the single boat analysis of Lauga \& Davis ${ }^{22}$ might be extended to the case of a flotilla of boats to examine how interaction with neighbouring boats can affect the propagation speed.

Since this paper has been concerned with the Stokes flow regime where the Lorentz reciprocal theorem is well-known, it should be mentioned that certain generalized reciprocal theorems relevant to Marangoni propulsion have been developed ${ }^{35}$. Their value lies in being able to use known analytical solutions to glean valuable information (e.g., the propulsion speed) in problems where the solution is not known. The analytical solutions found here may therefore facilitate a variety of new results to modified problems by feeding them in to suitable generalized reciprocal theorems.

It has been demonstrated how a mixed boundary value problem arising in shear flow over a model of superhydrophobic surfaces ${ }^{28,29}$ arises again in a model of a flotilla of Marangoni boats interacting through hydrodynamic effects caused by the diffusion of insoluble surfactant. The same mixed boundary value problem has also recently been found to have a surprising relevance to a temperature problem arising in thermocapillary flow over superhydrophobic surfaces ${ }^{36}$ where an external temperature gradient drives the flow. This mixed boundary value problem emerges in other problems too ${ }^{30,31}$; for example, the author has previously pointed out ${ }^{37}$ that hydrodynamic slip lengths over superhydrophobic surfaces have a completely different physical interpretation as the so-called blockage coefficients in ideal channel flows. The work here adds to the list of physical settings where this important class of mixed boundary value problems arises. 


\section{Appendix A: Complex variable formulation of Stokes flow}

Since the complex variable formulation of two-dimensional Stokes flow is not well known, this appendix gives a derivation of the relevant facts. The Stokes equations for a twodimensional velocity vector $\mathbf{u}=(u, v)$ are

$$
\mu \nabla^{2} \mathbf{u}=\nabla P, \quad \nabla \cdot \mathbf{u}=0
$$

On introducing a streamfunction related to the velocity field via (22), and on taking a curl of the first equation in (A1), the equations of Stokes flow are equivalent to

$$
\nabla^{4} \psi=0
$$

We will employ the notation $\mapsto$ to indicate a change to the "complex form" of some twodimensional vector quantity a, i.e.,

$$
\mathbf{a}=\left(\begin{array}{c}
a_{1} \\
a_{2}
\end{array}\right) \mapsto a_{1}+\mathrm{i} a_{2}
$$

With this convention, use of (22) implies

$$
\mathbf{u} \mapsto u+\mathrm{i} v=\frac{\partial \psi}{\partial y}-\mathrm{i} \frac{\partial \psi}{\partial x}=-\mathrm{i}\left[\frac{\partial \psi}{\partial x}+\mathrm{i} \frac{\partial \psi}{\partial y}\right]=-2 \mathrm{i} \frac{\partial \psi}{\partial \bar{z}}, \quad u-\mathrm{i} v=2 \mathrm{i} \frac{\partial \psi}{\partial z},
$$

where we have used (23), From (23) we can also write the vorticity $\omega=-\nabla^{2} \psi$ as

$$
\omega=-4 \frac{\partial^{2} \psi}{\partial z \partial \bar{z}}
$$

But, from (22),

$$
2 \mathrm{i} \psi=\bar{z} f(z)+g(z)-z \overline{f(z)}-\overline{g(z)}
$$

so that, from (A4), we find

$$
u+\mathrm{i} v=-f(z)+z \overline{f^{\prime}(z)}+\overline{g^{\prime}(z)}, \quad u-\mathrm{i} v=-\overline{f(z)}+\bar{z} f^{\prime}(z)+g^{\prime}(z) .
$$


If we use (A6) in (A5), we find

$$
\omega=-4 \frac{\partial^{2}}{\partial z \partial \bar{z}}\left[\frac{1}{2 \mathrm{i}}(\bar{z} f(z)+g(z)-z \overline{f(z)}-\overline{g(z)})\right]=-4\left(\frac{f^{\prime}(z)-\overline{f^{\prime}(z)}}{2 \mathrm{i}}\right)=-4 \operatorname{Im}\left[f^{\prime}(z)\right]
$$

The first equation in (A1) can be written, in complex form, as

$$
\nabla^{2} \mathbf{u}=\frac{1}{\mu} \nabla P \mapsto 4 \frac{\partial^{2}}{\partial \bar{z} \partial z}(u+\mathrm{i} v)=2 \frac{\partial}{\partial \bar{z}}\left(\frac{P}{\mu}\right)
$$

This can be integrated with respect to $\bar{z}$,

$$
\frac{P}{\mu}=2 \frac{\partial}{\partial z}(u+\mathrm{i} v)+F(z)
$$

where $F(z)$ is some, as yet unknown, analytic function. Relation (A10) can be written, using the first relation in (A7), as

$$
\frac{P}{\mu}=2\left(-f^{\prime}(z)+\overline{f^{\prime}(z)}\right)+F(z)
$$

Since the pressure $P$ is a real quantity we must pick $F(z)=4 f^{\prime}(z)$ :

$$
\frac{P}{\mu}=2\left(f^{\prime}(z)+\overline{f^{\prime}(z)}\right)=4\left[\frac{f^{\prime}(z)+\overline{f^{\prime}(z)}}{2}\right]=4 \operatorname{Re}\left[f^{\prime}(z)\right]
$$

Together, (A8) and (A12) imply the formula

$$
\frac{P}{\mu}-\mathrm{i} \omega=4 f^{\prime}(z)
$$

It is also clear that if

$$
\begin{aligned}
e_{11}+\mathrm{i} e_{12} & =\frac{1}{2}\left[\frac{\partial u}{\partial x}+\frac{\partial u}{\partial x}+\mathrm{i}\left(\frac{\partial u}{\partial y}+\frac{\partial v}{\partial x}\right)\right]=\frac{1}{2}\left[\frac{\partial u}{\partial x}-\frac{\partial v}{\partial y}+\mathrm{i}\left(\frac{\partial u}{\partial y}+\frac{\partial v}{\partial x}\right)\right] \\
& =\frac{1}{2}\left[\left(\frac{\partial}{\partial x}+\mathrm{i} \frac{\partial}{\partial y}\right)(u+\mathrm{i} v)\right]=\frac{\partial}{\partial \bar{z}}(u+\mathrm{i} v)=z \overline{f^{\prime \prime}(z)}+\overline{g^{\prime \prime}(z)} .
\end{aligned}
$$


In summary, so far we have

$$
\frac{P}{\mu}-\mathrm{i} \omega=4 f^{\prime}(z), \quad u+\mathrm{i} v=-f(z)+z \overline{f^{\prime}(z)}+\overline{g^{\prime}(z)}, \quad e_{11}+\mathrm{i} e_{12}=z \overline{f^{\prime \prime}(z)}+\overline{g^{\prime \prime}(z)} .
$$

Consider now a boundary of the fluid region. If $s$ denotes the arclength with the fluid to the left as the curve is traversed the unit tangent $\mathbf{t}$ in this direction has the complex form

$$
\mathbf{t} \mapsto \frac{d z}{d s}
$$

The complex form of the unit normal vector $\mathbf{n}$ out of the boundary and into the fluid is then

$$
\mathbf{n} \mapsto \mathrm{i} \frac{d z}{d s}
$$

To write the complex form of the fluid stress on the boundary first note that

$$
-p \mathbf{n} \mapsto-\left(\frac{4 \mu f^{\prime}(z)+4 \mu \overline{f^{\prime}(z)}}{2}\right) \mathrm{i} \frac{d z}{d s}
$$

It is also clear that

$$
e_{i j} n_{j}=\left(\begin{array}{c}
e_{11} n_{1}+e_{12} n_{2} \\
e_{21} n_{1}+e_{22} n_{2}
\end{array}\right) \mapsto e_{11} n_{1}+e_{12} n_{2}+\mathrm{i}\left(e_{21} n_{1}+e_{22} n_{2}\right)
$$

which can be written as

$$
\left(e_{11}+\mathrm{i} e_{12}\right) n_{1}+\mathrm{i}\left(e_{22}-\mathrm{i} e_{21}\right) n_{2}=\left(e_{11}+\mathrm{i} e_{12}\right)\left(n_{1}-\mathrm{i} n_{2}\right),
$$

where, in the last equality, we have used the fact that $e_{22}=-e_{11}$ and $e_{21}=e_{12}$. Hence

$$
e_{i j} n_{j} \mapsto\left(z \overline{f^{\prime \prime}(z)}+\overline{g^{\prime \prime}(z)}\right) \overline{\left(\mathrm{i} \frac{d z}{d s}\right)}
$$

It follows that

$$
-p n_{i}+2 \mu e_{i j} n_{j} \mapsto-2 \mu \mathrm{i}\left(f^{\prime}(z)+\overline{f^{\prime}(z)}\right) \frac{d z}{d s}-2 \mu \mathrm{i}\left(z \overline{f^{\prime \prime}(z)}+\overline{g^{\prime \prime}(z)}\right) \frac{d \bar{z}}{d s}
$$


Hence

$$
-p n_{i}+2 \mu e_{i j} n_{j} \mapsto-2 \mu \mathrm{i} \frac{d H}{d s}, \quad H \equiv f(z)+z \overline{f^{\prime}(z)}+\overline{g^{\prime}(z)} .
$$

\section{REFERENCES}

${ }^{1}$ A. Zöttl \& H. Stark, Emergent behavior in active colloids, J. Phys.: Condens. Matter, 28, 253001, (2016).

${ }^{2}$ N. J. Suematsu \& S. Nakata, Evolution of self-propelled objects: from the viewpoint of nonlinear science, Chem. Eur. J., 24, 6308 -6324, (2018).

${ }^{3}$ S. Nakata, M. Nagayama, H. Kitahata, N. J. Suematsu, and T. Hasegawa, Physicochemical design and analysis of self- propelled objects that are characteristically sensitive to environments, Phys. Chem. Chem. Phys., 17, 10326 (2015).

${ }^{4}$ L. Botto, E. P. Lewandowski, M. Cavallaro Jr. \& K. J. Stebe, Capillary interactions between anisotropic particles, Soft Matter, 8, 9957, (2012).

${ }^{5}$ J. W. M. Bush \& D. L. Hu, Walking on water: biolocomotion at the interface, Ann. Rev. Fluid Mech., 38, 339-369, (2006).

${ }^{6}$ K. Dietrich, N. Jaensson, I. Buttinoni, G. Volpe \& L. Isa, Microscale Marangoni surfers, Phys. Rev. Lett., 125, 098001, (2020).

${ }^{7}$ A. Würger, Thermally driven Marangoni surfaces, J. Fluid Mech., 752, 589-601, (2014).

${ }^{8}$ E. Chevallier, A. Mamane, H. A. Stone, C. Tribet, F. Lequeuxa and C. Monteux, Pumpingout photo-surfactants from an air-water interface using light, Soft Matter, 7, 7866, (2011).

${ }^{9}$ A. Diguet, R-M. Guillermic, N. Magome, A. Saint-Jalmes, Y. Chen, K. Yoshikawa and D. Baigl, Photomanipulation of a droplet by the chromocapillary effect, Angew. Chem. Int. Ed., 48, 9281 -9284, (2009).

${ }^{10}$ S.N. Varanakkottu, S. D. George, T. Baier, S. Hardt, M. Ewald, and M. Biesalski, Particle manipulation based on optically controlled free surface hydrodynamics, Angew. Chem. Int. Ed., 52, $7291-7295,(2013)$.

${ }^{11}$ S. Nakata, M. Nagayama, H Kitahata, N. J. Suematsu \& T. Hasegawa, Physicochemical design and analysis of self- propelled objects that are characteristically sensitive to environments, Phys. Chem. Chem. Phys., 17, 10326, (2015).

${ }^{12}$ M. I Kohira, Y. Hayashima, M. Nagayama \& S. Nakata, Synchronized self-motion of two camphor boats, Langmuir, 17, 7124-7129, (2001). 
${ }^{13}$ T. Toyota, N. Maru, M. M. Hanczyc, T. Ikegami \& T. Sugawara, Self-propelled oil droplets consuming "fuel" surfactant, J. Am. Chem. Soc., 131, 5012-5013, (2009).

${ }^{14}$ N. J. Suematsu, S. Nakata, A. Awazu \& H. Nishimori, Collective behavior of inanimate boats, Phys. Rev. E, 81, 056210, (2010)

${ }^{15}$ S. Soh, K. J. M. Bishop \& B. A. Gryzbowski, Dynamic self-assembly in ensembles of camphor boats, J. Phys. Chem. B, 112, 10848-10853, (2008).

${ }^{16}$ K. Ikeda, S-I Ei, M. Nagayama, M. Okamoto \& A. Tomoeda, Reduced model of a reactiondiffusion system for the collective motion of camphor boats, Phys. Rev. E, 99, 062208, (2019).

${ }^{17}$ E. Heisler, N. J. Suematsu, A. Awazu, and H. Nishimori, Collective motion and phase transitions of symmetric camphor boats, J. Phys. Soc. Jpn., 81 074695, (2012).

${ }^{18}$ H. Kitahata, S Hiromatsu, Y. Doi, S. Nakata \& M. R. Islam, Self-motion of a camphor disk coupled with convection, Phys. Chem. Chem. Phys, 6, 2409-2414, (2004).

${ }^{19}$ Y. S. Ikura, R. Tenno, H. Kitahata, N J. Suematsu \& S. Nakata, Suppression and regeneration of camphor-driven Marangoni flow with the addition of sodium dodecyl sulfate, $J$. Phys. Chem. B, 116, 992-996, (2012).

${ }^{20}$ H. Kitahata \& N. Yoshinaga, Effective diffusion coefficient including the Marangoni effect, J. Chem. Phys., 148, 134906, (2018).

${ }^{21}$ T. Bickel, Spreading dynamics of reactive surfactants driven by Marangoni convection, Soft Matter, 15, 3644, (2019).

${ }^{22}$ E. Lauga \& A. M. J. Davis, Viscous Marangoni propulsion, J. Fluid Mech., 705, 120-133, (2012).

${ }^{23}$ D. Halpern \& A. L. Frenkel, Destabilization of a creeping flow by interfacial surfactant: linear theory extended to all wavenumbers, J. Fluid Mech., 485, 191-220, (2003).

${ }^{24} \mathrm{M}$. Siegel, Influence of surfactant on rounded and pointed bubbles in two-dimensional Stokes flow, SIAM J. Appl. Math., 59, 1998-2027, (1999).

${ }^{25}$ D. G. Crowdy, Surfactant-induced stagnant zones in the Jeong-Moffatt free surface Stokes flow problem, Phys. Fluids., 25, 092104, (2013).

${ }^{26}$ D. G. Crowdy \& S. Tanveer, A theory of exact solutions for plane viscous blobs, J. Nonlin. Sci., 8, 261-279, (1998).

${ }^{27}$ W. E. Langlois and M. O. Deville, Slow viscous flows, Second Edition, Springer, (2014).

${ }^{28}$ J. R. Philip, Flows satisfying mixed no-slip and no-shear conditions, J. Appl. Math. Phys. 
(ZAMP), 23, 353-372, (1972).

${ }^{29}$ D. G. Crowdy, Frictional slip lengths for unidirectional superhydrophobic grooved surfaces, Phys. Fluids, 23, 072001, (2011).

${ }^{30}$ B. Ya. Moizhes, Averaged electrostatic boundary conditions for metallic meshes, Zh. Tekh. Fiz., 25, 167-176, (1955).

${ }^{31} \mathrm{H}$. Hasimoto, On the flow of a viscous fluid past a thin screen at small Reynolds number, J. Phys. Soc. Japan, 13, 633-639, (1958).

${ }^{32}$ D. G. Crowdy, Solving problems in multiply connected domains, NSF-CBMS Regional Conference Series in Applied Mathematics, Society for Industrial and Applied Mathematics, Philadelphia, (2020).

${ }^{33}$ D. G. Crowdy, Perturbation analysis of subphase gas and meniscus curvature effects for longitudinal flows over superhydrophobic surfaces, J. Fluid Mech., 822, 307-326, (2017).

${ }^{34}$ T. Kirk, G. Karamanis, D. G. Crowdy \& M. Hodes, Thermocapillary stress and meniscus curvature effects on slip lengths in ridged microchannels, J. Fluid Mech., 894, A15, (2020).

${ }^{35} \mathrm{H}$. Masoud \& H. A. Stone, A reciprocal theorem for Marangoni propulsion, 741, R4, (2014).

${ }^{36}$ E. Yariv \& D. G. Crowdy, Thermocapillary flow between grooved superhydrophobic surfaces: transverse temperature gradients, J. Fluid Mech., 871, 775-798, (2019).

${ }^{37}$ D. G. Crowdy, Frictional slip lengths and blockage coefficients, Phys. Fluids, 23, 091703, (2011). 